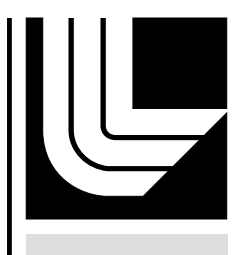

LAW RENCE LIVERMORE N A TIO N A L LABORATORY

\title{
Gas Detector LCLS Engineering Specifications Document
}

Stefan Hau-Riege, Paul Pax

February 16, 2007 
This document was prepared as an account of work sponsored by an agency of the United States Government. Neither the United States Government nor the University of California nor any of their employees, makes any warranty, express or implied, or assumes any legal liability or responsibility for the accuracy, completeness, or usefulness of any information, apparatus, product, or process disclosed, or represents that its use would not infringe privately owned rights. Reference herein to any specific commercial product, process, or service by trade name, trademark, manufacturer, or otherwise, does not necessarily constitute or imply its endorsement, recommendation, or favoring by the United States Government or the University of California. The views and opinions of authors expressed herein do not necessarily state or reflect those of the United States Government or the University of California, and shall not be used for advertising or product endorsement purposes.

This work was performed under the auspices of the U.S. Department of Energy by University of California, Lawrence Livermore National Laboratory under Contract W-7405-Eng-48. 


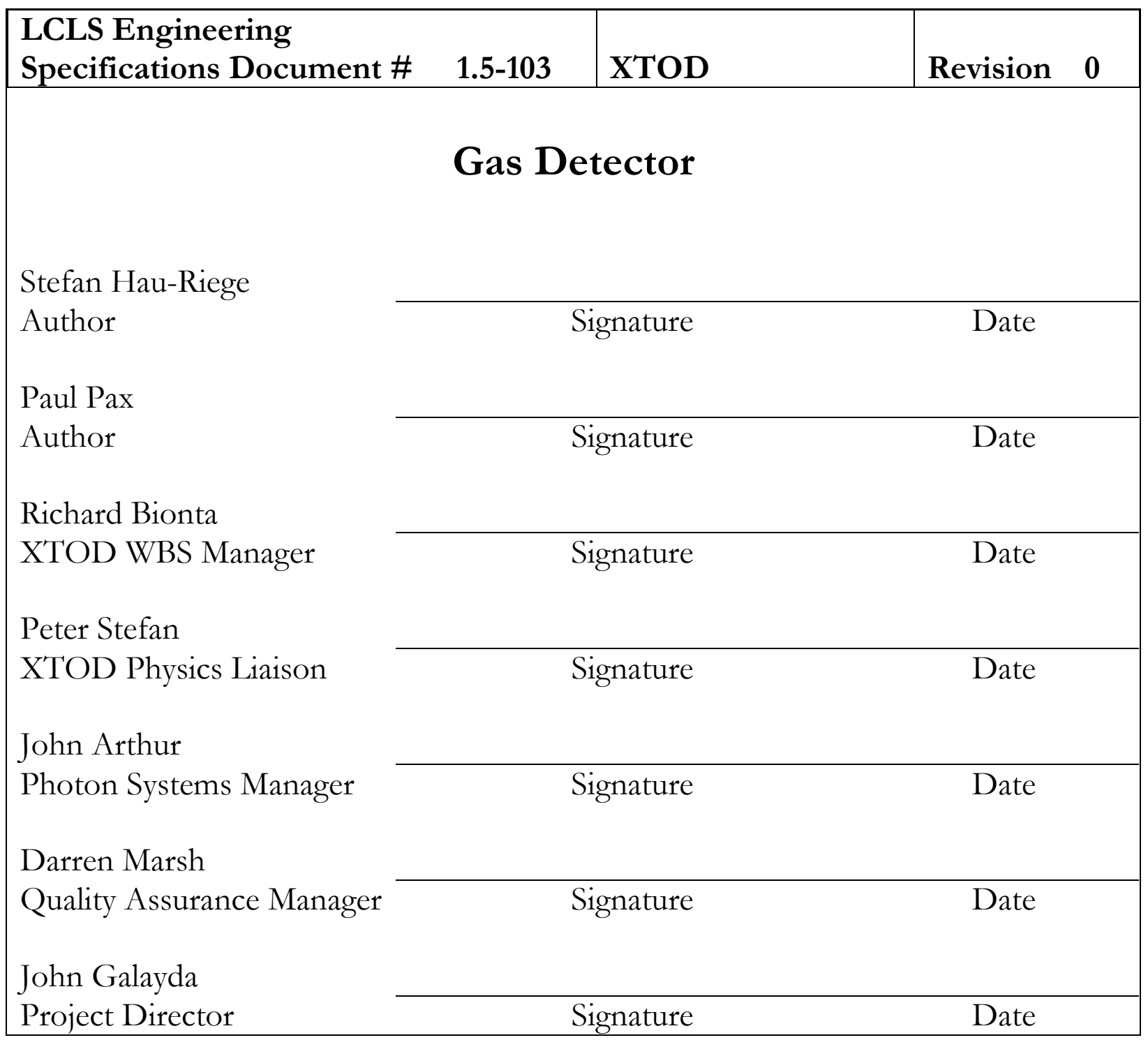

\section{Summary:}

This document analyzes and summarizes the physics behind the operation of the Gas Detectors and derives their consequent engineering design.

Change History Log

\begin{tabular}{|l|l|l|l|}
\hline $\begin{array}{l}\text { Rev } \\
\text { Number }\end{array}$ & $\begin{array}{l}\text { Revision } \\
\text { Date }\end{array}$ & Sections Affected & Description of Change \\
\hline 000 & $2007 / 2 / 9$ & All & Initial Version \\
\hline & & & \\
\hline
\end{tabular}




\section{Disclaimer}

This document was prepared as an account of work sponsored by an agency of the United States Government. Neither the United States Government nor the University of California nor any of their employees, makes any warranty, express or implied, or assumes any legal liability or responsibility for the accuracy, completeness, or usefulness of any information, apparatus, product, or process disclosed, or represents that its use would not infringe privately owned rights. Reference herein to any specific commercial product, process, or service by trade name, trademark, manufacturer, or otherwise, does not necessarily constitute or imply its endorsement, recommendation, or favoring by the United States Government or the University of California. The views and opinions of authors expressed herein do not necessarily state or reflect those of the United States Government or the University of California, and shall not be used for advertising or product endorsement purposes.

\section{Auspices Statements}

This work was performed under the auspices of the U.S. Department of Energy by University of California, Lawrence Livermore National Laboratory under Contract W-7405-Eng-48 and also supported in part by the DOE Contract DE-AC0276SF00515. This work was performed in support of the LCLS project at SLAC. 


\section{Executive Summary:}

There are two Gas Detectors, located upstream and downstream of the FEL attenuation materials, which provide a non-intrusive measure of the FEL pulse energy in the fundamental, in real-time, on a pulse-by-pulse basis. The FEL operators and the users will use this information to monitor the performance of the FEL and the Attenuator and to crosscalibrate other detectors. The Gas Detectors measure the FEL pulse energy by measuring the fluorescence induced in a small volume of $\mathrm{N}_{2}$ gas by the passage of the FEL. 


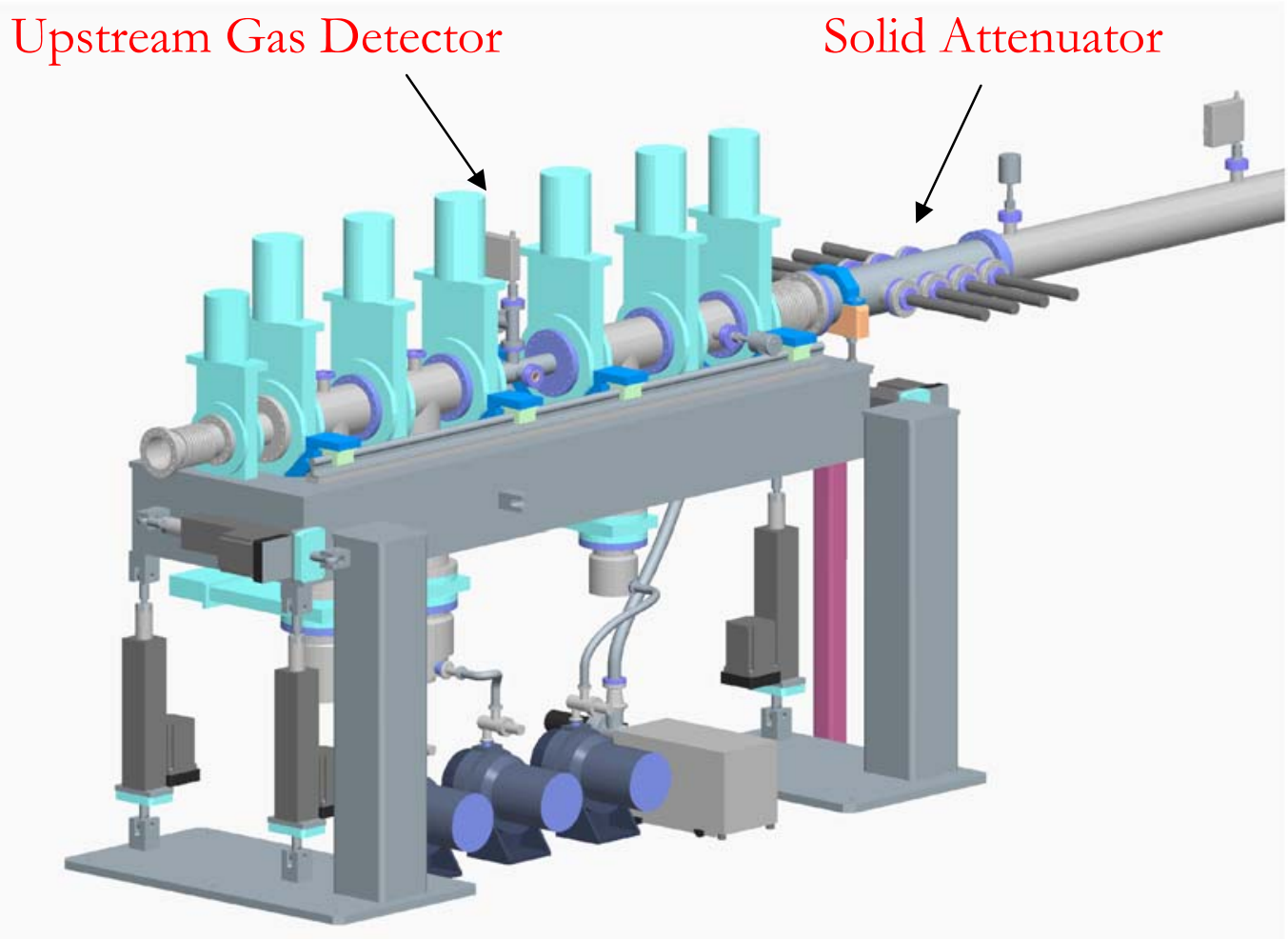

Figure 1: System configuration for the Gas Detector

\section{System Requirements}

LCLS Physics Requirements Document for the Gas Detector [1] presents the fundamental requirements for the system; these requirements will not be reiterated here.

\section{System Description}

\section{A. Overview}

The two Gas Detectors are located in the Front End Enclosure (FEE) and are integrated into the upstream and downstream differential pumping sections of the Gas Attenuator [2]. A system configuration, showing the differential pumping stages shared with the Gas Attenuator, is shown in Figure 1. The Gas Detectors measure the FEL pulse energy, pulse-to-pulse and non-intrusively, by measuring the fluorescence induced in a small volume of $\mathrm{N}_{2}$ gas by the passage of the FEL.

Figure 2 shows a schematic of the Gas Detector with its principle subsystems. The main detector vessel is a section of cylindrical pipe filled with $\mathrm{N}_{2}$ gas at pressures between 0.1 Torr to 2 Torr, depending upon the photon energy of the FEL fundamental. The FEL radiation freely enters and 
exits the pressurized cylinder through a series of $3 \mathrm{~mm}$ diameter apertures in the differential pumping sections at each end. A small fraction of the FEL radiation interacts with the atoms in the gas, primarily through photoionization, producing photoelectrons whose energies are a substantial fraction of the initial FEL photon energy. The photoelectrons are confined by the magnetic field to helical trajectories about the beam axis. As the photoelectrons traverse the gas, they produce secondary electrons which, in turn, excite $\mathrm{N}_{2}$ molecular states that emit optical radiation, primarily in the near-UV band between 300 to $430 \mathrm{~nm}$. The UV radiation emitted by the gas is sampled by the three near-UV detectors that view the central portion of the excited gas. The three detectors, a photodiode (PD), an avalanche photodiode (APD) and a photomultiplier tube (PMT), cover different near-UV light levels, and allow the Gas Detector to operate over a range of signal levels covering 4 orders of magnitude.

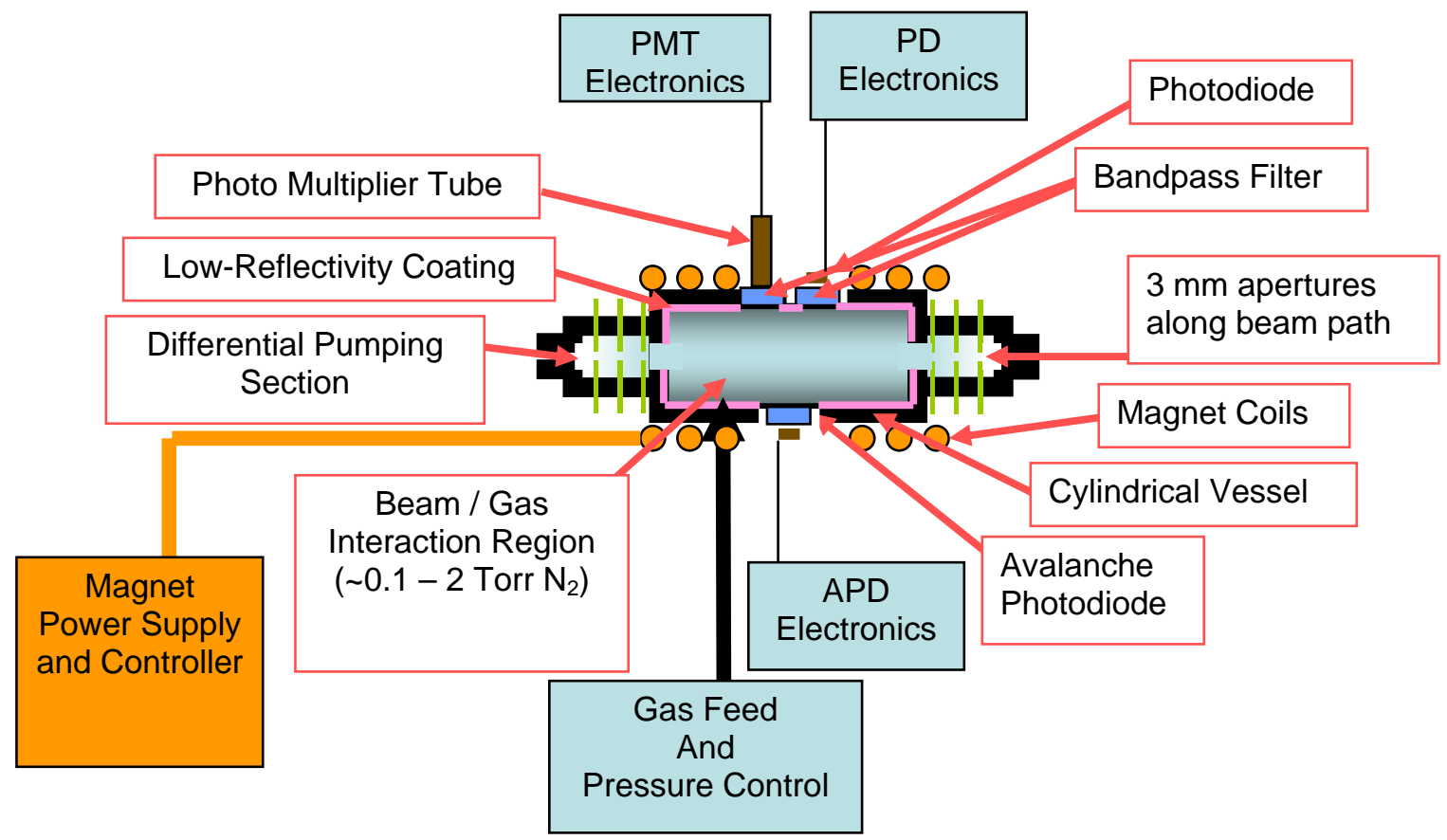

Figure 2: Schematics of the Gas Detector indicating the principle subsystems.

The primary photoelectrons loose energy in the gas through inelastic scattering with the nitrogen molecules. A large fraction of them eventually exit the ends of the chamber and strike the solid parts of one of the $3 \mathrm{~mm}$ apertures. This may potentially create light that could strike the detectors and mask the signal from the gas. Therefore, there are several features built into the detector to inhibit light that does not originate from the gas from then striking the detectors. The photo detectors are recessed, to block the direct path from the solid parts of the $3 \mathrm{~mm}$ apertures to the active areas of the detectors. The inside surface of the cylindrical chamber is grooved to deflect photons emitted from the solid parts of the $3 \mathrm{~mm}$ apertures that would otherwise strike the detector after a single bounce. The band pass filters in front of the detectors pass only light in the near-UV band. And finally, the cylinder is made of stainless steel with a coating that has less than $20 \%$ reflectivity under normal incidence in the near-UV band. 


\section{B. Operating Principle}

The LCLS beam entering the Gas Detector consists of a spontaneous background signal from the undulator, of which a small bandwidth (the fundamental) is amplified by the FEL process. The spontaneous radiation spans an energy range up to several $\mathrm{MeV}$, whereas the fundamental lies between $826 \mathrm{eV}$, what we call "the soft x-ray case" from now on, and $8267 \mathrm{eV}$, called "the hard x-ray case".

Energy Absorption: A small fraction of the LCLS beam (for a $30 \mathrm{~cm}$-long gas cell, 2.4\% of soft x-ray photons at 0.1 Torr, and $0.06 \%$ of hard $\mathrm{x}$-ray photons at 2 Torr) is absorbed through $\mathrm{K}$-shell photoionization. Saturation effects are not a concern since the fraction of $\mathrm{K}$-shell-ionized $\mathrm{N}_{2}$ molecules is less than $0.4 \%$. The energy of the ejected photoelectrons is $\left(\mathrm{E}_{\text {photon }}-\mathrm{E}_{\text {ion }}\right)$, where $\mathrm{E}_{\text {photon }}$ is the photon energy, and $\mathrm{E}_{\mathrm{ion}} \sim 0.4 \mathrm{keV}$ is the $\mathrm{K}$-shell electron ionization energy. Within a few femtoseconds, the ionized nitrogen relaxes by emitting an Auger electron of energy $\sim 0.4 \mathrm{keV}$. The photo- and Auger-electrons deposit their energy through electron-impact ionization and electronic excitation while passing through the gas, until they are thermalized or reach the detector walls. To maximize the fraction of energy deposited into the $\mathrm{N}_{2}$, we apply a weak axial magnetic field (40 Gauss for the soft x-ray case and 200 Gauss for the hard x-ray case). Representative trajectories of the photo- and Auger-electrons at these magnetic fields are shown in Figure 3. Increasing the magnetic field further does not improve the signal significantly, since most of the electrons eventually exit the ends of the chamber and strike one of the solid parts of the $3 \mathrm{~mm}$ apertures.

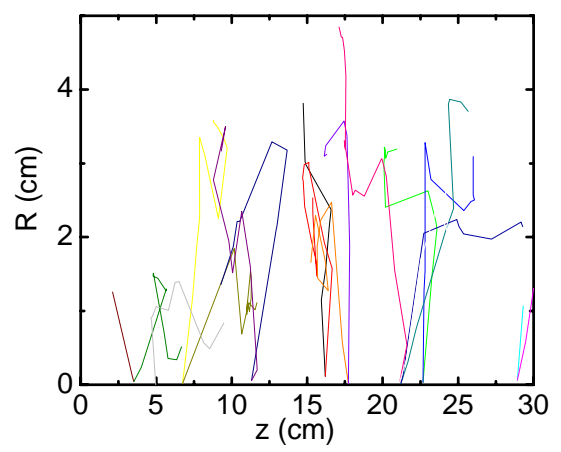

(a)

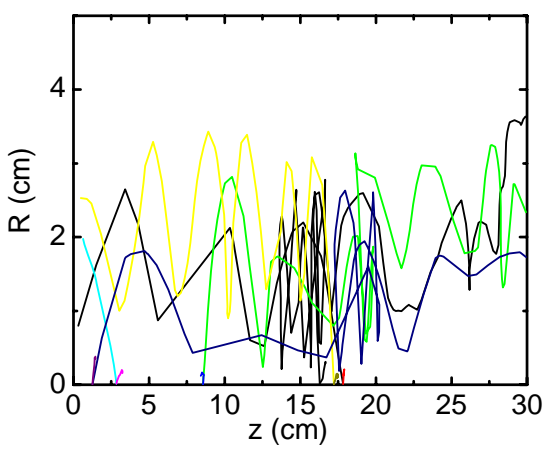

(b)

Figure 3: Typical photo- and Auger-electron trajectories in $\mathrm{N}_{2}$ for (a) $0.83 \mathrm{keV}$ x-rays at 0.1 Torr and (b) $8.3 \mathrm{keV}$ x-rays at 2 Torr. 
Nitrogen Fluore scence Spectrum

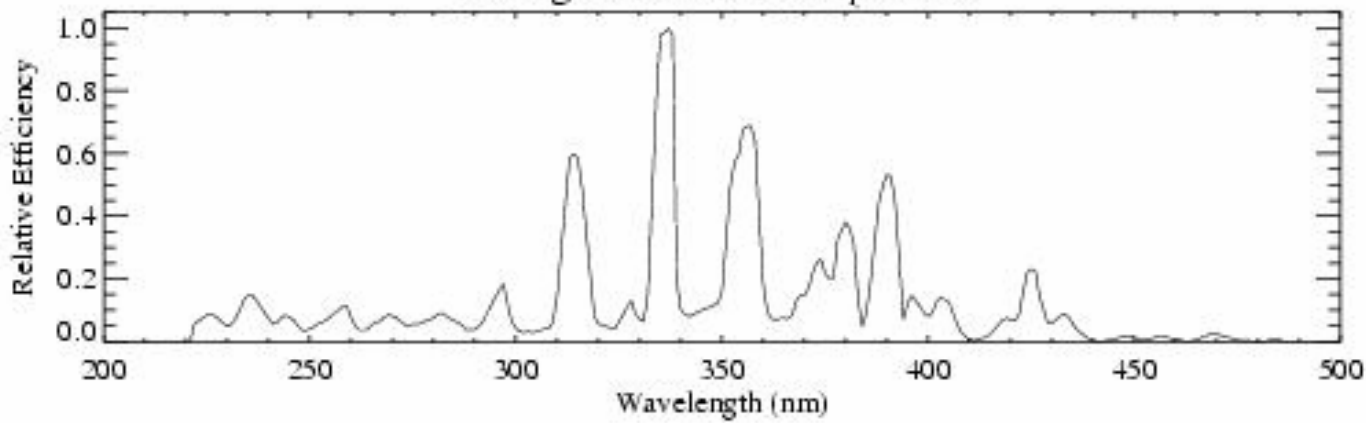

Figure 4: Nitrogen fluorescence spectrum.

$\mathrm{N}_{2}$ Fluorescence: The excited nitrogen gas relaxes through the emission of near-UV photons within 20 -30 ns [3]. The emission spectrum is shown in Figure 4 [4]. Figure 5 shows the near-UV photoluminescence signal based on experimental data from the field of ultra-high-energy cosmic rays [3]. That specific dataset was collected for $0.83 \mathrm{MeV}$ electrons entering a $\mathrm{N}_{2}$ gas cell. It has been shown that the fluorescence yield per deposited energy depends only weakly on the energy of the incoming electron, see Figure 6 [5]. The amount of near-UV radiation should correlate to the intensity of the LCLS beam: For $\mathrm{N}_{2}$ pressures between $0.1-2$ Torr, the fluorescence yield of $\mathrm{N}_{2}$ is $\sim 1.3 \mathrm{UV}$ photons per deposited energy in $\mathrm{keV}[3,5]$.
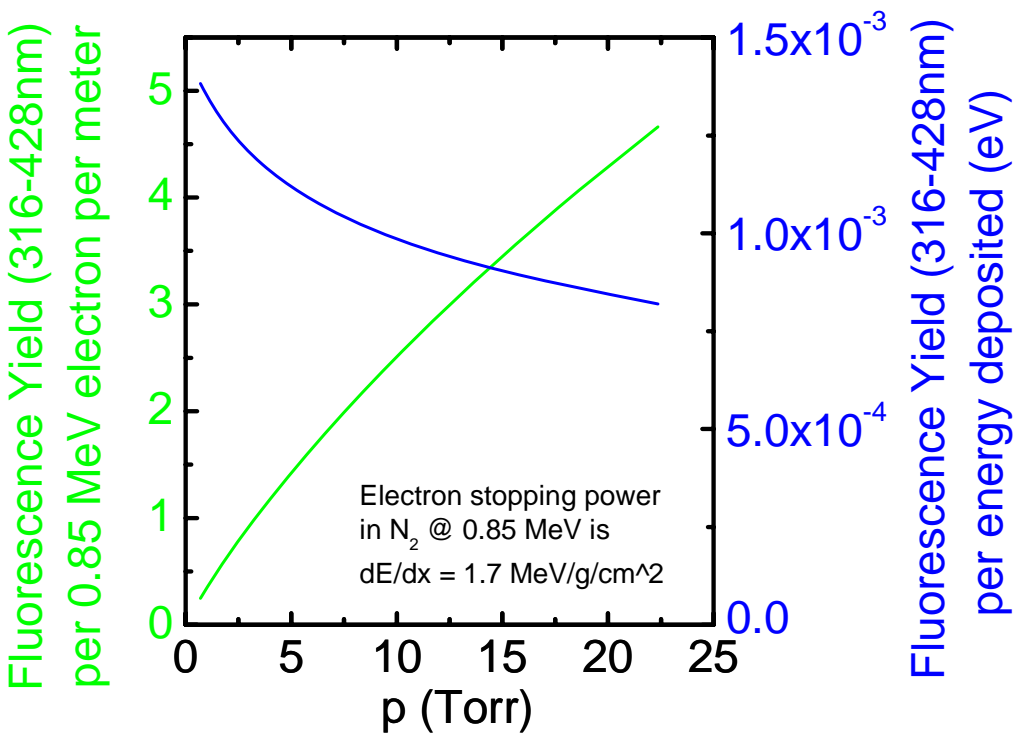

Figure 5: Near-UV photon yield due to excitations by $0.85 \mathrm{MeV}$ electrons in $\mathrm{N}_{2}$ as a function of $\mathrm{N}_{2}$ pressure. 


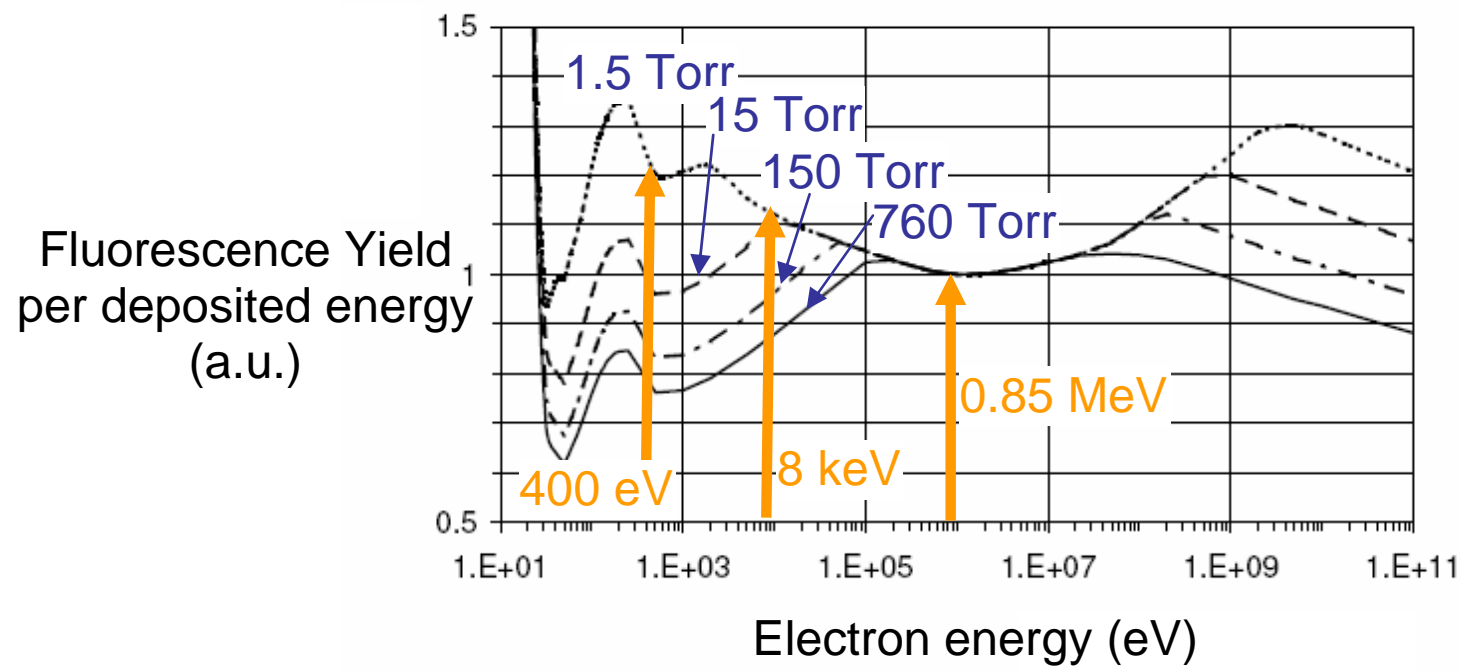

Figure 6: Near-UV photon yield in $\mathrm{N}_{2}$ irradiated by electrons as a function of electron energy and $\mathrm{N}_{2}$ pressure.

Signal: We estimated the signal entering a detector window of size $1 \mathrm{~cm}^{2}$ through Monte Carlo simulations. For a soft x-ray photon beam with pulse energy $0.1 \mu \mathrm{J}, 1.8 \times 10^{4}$ photons per $\mathrm{cm}^{2}$ reach the detector port, whereas for a hard x-ray photon beam with pulse energy $1 \mu \mathrm{J}, 2.2 \times 10^{3}$ photons per $\mathrm{cm}^{2}$ reach the detector port, assuming the detector wall does not reflect near-UV photons. If the detectors are recessed, the actual signal is reduced by a factor of 2 to 3 .

Configuration: The interaction of ions and electrons with the chamber walls is not very well understood; we designed the Gas Detector to minimize the number of photons generated during these interactions from entering the detector: (i) The detector windows should be recessed to prevent photons, generated by ions and electrons hitting the apertures at the detector ends, from entering the detector. (ii) The chamber walls are non-reflecting in the UV range, e.g. by using a grooved, stainless steel vessel.

Noise (Response to Spontaneous Radiation): In addition to the FEL fundamental, the spontaneous radiation will also deposit energy in the detector gas through Compton scattering and absorption. We have estimated the energy deposited by these processes to be $2.5 \mathrm{~nJ}$ for the soft x-ray case, and $40.5 \mathrm{~nJ}$ for the hard x-ray case. FEL fundamental pulse energies of $0.14 \mu \mathrm{J}$ and $106 \mu \mathrm{J}$ (for the softand hard-x-ray cases, respectively) would deposit equivalent energy in the gas, so that the FELfundamental-equivalent fluctuations would be $1 \mathrm{~nJ}$ and $460 \mathrm{~nJ}$, respectively. For comparison, the lowest FEL-fundamental pulse energies that are required to be detected are 100 and $1000 \mathrm{~nJ}$, respectively [1]. 

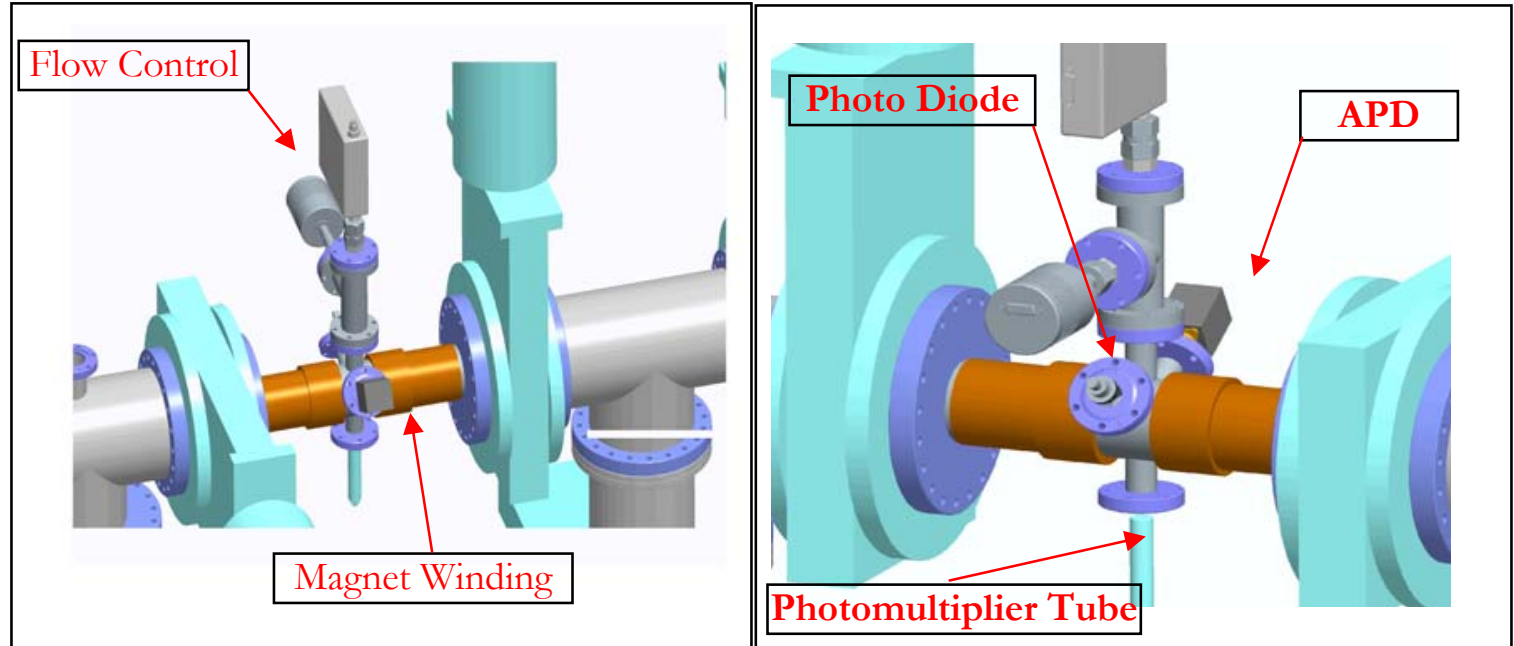

Figure 7: Configuration of the Gas Detector System.

\section{Subsystems}

\section{Magnet}

To confine the primary photoelectrons, an axial magnetic field of at least 200 Gauss is required. A room-temperature solenoidal winding is desirable. Figure 7 shows a schematic of the Gas Detector with the solenoidal winding illustrated. The winding is interrupted in the middle of the detector to allow for the detector ports. To overcome the inhomogenities in the resulting magnetic field, additional windings are installed just up- and downstream of the detector ports. In this configuration, the power consumption is smaller than $100 \mathrm{~W}$, with an operating current below $20 \mathrm{~A}$. Within the detector port, which creates a gap of about $50 \mathrm{~mm}$, the magnetic field stays above 200 Gauss. The field uniformity requirements of $<-10 \% /+20 \%$ for spatial variation (for homogeneous electron energy deposition) and $< \pm 1 \%$ for temporal variation (for $< \pm 1 \%$ pulse-to-pulse reproducibility of the Gas Detector signal [1]) are met. The power supply will be controlled by the EPICS system.

\section{Optics}

The geometry of the Gas Detector chamber and the surface properties of its inside wall will have an effect on the signal levels reaching the detectors. To avoid uncertainties in the calibration due to geometry and aging of surface properties, it is desirable to minimize any indirect paths from the fluorescence source to the photodetector. For this purpose, the inside of the cylinder will be treated to minimize its reflectivity, and light traps may be constructed opposite the photodetectors. Such a light trap enhances absorption by its geometric configuration. A simple example of a light trap is a wedged cavity or pipe, with moderately-absorbing surfaces. Any light entering the wedge will require multiple reflections in order to leave it; if the number of reflections is high enough, variations in the wall reflectivity will have only a small effect on indirect signals reaching the photodetector. 


\section{Near-UV Photon Detectors}

The Gas Detector monitors the fluorescence signal induced by the interaction of the FEL beam with the nitrogen gas. The fluorescence signal, in turn, will be measured by a PMT, an APD, and a silicon PD, used in conjunction with bandpass filters.

Use of multiple detector types will allow matching to a range of signal levels, and enhance cross calibration. PMTs are well known for their sensitivity and dynamic range, and are therefore a natural choice for this application. However, there is some concern about their sensitivity to magnetic fields, as well as their absolute calibration. PDs and APDs are solid-state devices with well characterized responses, but typically suffer from a higher noise level. To cover the expected range of signal levels, the PMT and APD gains must be varied and this should be controllable remotely. Together, the PMT, APD, and PD will provide the sensitivity and dynamic range required for the Gas Detector.

Optical noise sources include photons produced by the interaction of electrons and nitrogen ions with the chamber walls, light generated at the downstream target interactions, and the spontaneous radiation generated upstream. Optical bandpass filters will be used to limit the signal to the band produced by the nitrogen fluorescence, and will be installed in the vacuum window. It is expected that interactions of the electrons and ions with the chamber walls will be concentrated at the chamber end caps, due to the lack of longitudinal confinement. To further suppress this noise source, the detectors will be recessed into the chambers walls, preventing any direct line-of-sight to the end caps.

\section{Light Source}

Gas Detector will be calibrated using the Total Energy Measurement System (TEM) [6]. We will also include an additional, auxiliary calibration system with the Gas Detector that is completely internal to it. This calibration system will consist of UV Light Emitting Diode (UVLED) sources, whose output will be well-characterized in conjunction with the detectors. While this calibration system will be secondary to the TEM, it will provide a convenient way to check performance and troubleshoot, in that it can be used independently of the TEM, and of the FEL itself.

\section{Read-Out Electronics}

The near-UV signal is expected to be 20 to $30 \mathrm{~ns}$ in duration. At a sampling rate of $1 \mathrm{~ns}$, corresponding to $1 \mathrm{GHz}$, it is expected that the signal can clearly be detected and distinguished from possible prompt-pulse signals that precede the actual FEL pulse. It is further desirable to sample the signal for a significant longer time, up to 100's of ns, in order to detect slower signals that could possibly be used to analyze the FEL output. The use of a shaping amplifier is not preferable, since it masks the actual detector signal.

Each detector system (PD, APD, and PMT) has its associated signal-processing electronics and control.

The PMT detector

The PMT detector signal-processing electronics consists of

1) $\quad$ A PMT

2) High Voltage supply that allows remote setting of the PMT voltage (gain) 
3) High speed Analog-to-Digital converter (ADC)

4) EPICS-compatible controller

Elements of the PMT signal processing electronics will be off-the-shelf (OTS) where possible.

The APD detector

The APD detector signal-processing electronics consists of

1) An APD

2) APD bias and high voltage circuitry

3) APD pre-amp

4) Analog-to-Digital converter (ADC)

5) EPICS-compatible controller

Elements of the APD signal processing electronics will be OTS where possible.

The PD detector

The PD detector signal-processing electronics consists of

1) $\quad \mathrm{A} P D$

2) Bias supply voltage

3) Photodiode pre-amp

4) Gated integrator (if needed)

5) Analog-to-Digital converter (ADC)

6) EPICS-compatible controller

Elements of the PD signal processing electronics will be OTS where possible.

\section{Mechanical \& Vacuum}

The gas detector system will be integrated into the differential pumping stages of the Gas/Solid Attenuator System. The system configuration is illustrated in Figure 1. A description of the XTOD Gas/Solid Attenuator System can be found in the ESD report [2].

\section{Controls}

The design of the control system for the LCLS XTOD Gas Detector will be based on the design of the LCLS XTOD tunnel vacuum system and the LCLS XTOD Attenuator System. The Gas Detector and the Gas Attenuator vacuum and gas-flow controls are treated as a single entity, where a PLC (Programmable Logic Controller) provides basic interlocking and EPICS sequences choreograph transitions between major states (vacuum-all-through; detectors-only; detectors-andattenuator; etc). The Gas Detector processed measurements will be fed to experiments (XES/LUSI) and elsewhere via a timing system (per pulse) as well as via Process Variables (slow). The PLC reports to MPS for critical items The control system's basic design and specifically the vacuum control area are described in Ref. [2]. We aim for full compatibility with the global control system, EPICS. 


\section{Additional System Requirements and Derived Specifications}

To maximize the fraction of energy deposited into the $\mathrm{N}_{2}$, we apply a weak axial magnetic field (40 Gauss for the soft x-ray case and 200 Gauss for the hard x-ray case). The field uniformity requirements are $<-10 \% /+20 \%$ for spatial variation and $< \pm 1 \%$ for temporal variation.

The fluorescence signal will be measured by a PMT, an APD, and a silicon PD, used in conjunction with bandpass filters. The readout electronics should measure the near-UV signal, that is expected to be 20 to $30 \mathrm{~ns}$ in duration, at a sampling rate of $1 \mathrm{~ns}$, for 100 's of ns.

\section{Approach Forward}

Coherent and incoherent scattering of the fundamental and spontaneous radiation has recently been identified as a possible important noise source. Further modeling is required to estimate the extent of this issue. We will also perform initial experiments at SSRL to determine the effect of the scattered fundamental on the detector signal by measuring the polarization-dependence of the scattered signal.

\section{References}

[1] "Physics Requirements for the XTOD Gas Detector System", LCLS Physics Requirements Document \#1.5-008.

[2] "XTOD Attenuator", LCLS Engineering Specifications Document \#1.5-102.

[3] M. Nagano, K. Kobayakawa, N. Sakaki, and K. Ando, "Photon yields from nitrogen gas and dry air excited by electrons”, Astrophys. Part. 22, 235 (2004).

[4] A.N. Brunner, Ph.D. Thesis, Cornell University (1967).

[5] F. Blanco and F. Arqueros, "The role of secondary electrons in some experiments determining the fluorescence emission from nitrogen $C^{3} \Pi_{u}$ levels", Phys. Lett. A 345, 355 (2005).

[6] "XTOD Total Energy Measurement System", LCLS Physics Requirements Document \#1.5-009. 